\title{
BMJ Open Acceptability of OP/Na swabbing for SARS-CoV-2: a prospective observational cohort surveillance study in Western Australian schools
}

\begin{abstract}
Hannah M Thomas (D) , , Marianne J Mullane, ${ }^{1,2}$ Sherlynn Ang, ${ }^{1}$ Tina Barrow, ${ }^{1}$ Adele Leahy, ${ }^{1,2}$ Alexandra Whelan, ${ }^{1,2}$ Karen Lombardi, ${ }^{1,3}$ Matthew Cooper, ${ }^{1}$ Paul G Stevenson, ${ }^{1}$ Leanne Lester, ${ }^{4}$ Andrea Padley, ${ }^{5}$ Lynn Sprigg, ${ }^{5}$ David Speers, ${ }^{4,6}$ Adam J Merritt, ${ }^{6}$ Juli Coffin,, ${ }^{1,4}$ Donna Cross, , ${ }^{1,4}$ Peter Gething, ${ }^{1,7}$ Asha C Bowen ${ }^{1,2,4}$
\end{abstract}

To cite: Thomas HM, Mullane MJ, Ang S, et al. Acceptability of $\mathrm{OP} / \mathrm{Na}$ swabbing for SARS-CoV-2: a prospective observational cohort surveillance study in Western Australian schools. BMJ Open 2022;12:e055217. doi:10.1136/ bmjopen-2021-055217

- Prepublication history for this paper is available online. To view these files, please visit the journal online (http://dx.doi. org/10.1136/bmjopen-2021055217).

Received 08 July 2021 Accepted 28 0ctober 2021

\section{Check for updates}

(c) Author(s) (or their employer(s)) 2022. Re-use permitted under CC BY-NC. No commercial re-use. See rights and permissions. Published by BMJ.

For numbered affiliations see end of article.

\section{Correspondence to} Dr Hannah M Thomas; hannah.thomas@telethonkids. org.au

\section{ABSTRACT}

Objectives When the COVID-19 pandemic was declared, Governments responded with lockdown and isolation measures to combat viral spread, including the closure of many schools. More than a year later, widespread screening for SARS-CoV-2 is critical to allow schools and other institutions to remain open. Here, we describe the acceptability of a minimally invasive COVID-19 screening protocol trialled by the Western Australian Government to mitigate the risks of and boost public confidence in schools remaining open. To minimise discomfort, and optimise recruitment and tolerability in unaccompanied children, a combined throat and nasal (OP/Na) swab was chosen over the nasopharyngeal swab commonly used, despite slightly reduced test performance.

Design, setting and participants Trialling of OP/ Na swabbing took place as part of a prospective observational cohort surveillance study in 79 schools across Western Australia. Swabs were collected from 5903 asymptomatic students and 1036 asymptomatic staff in 40 schools monthly between June and September 2020.

Outcome measures PCR testing was performed with a two-step diagnostic and independent confirmatory PCR for any diagnostic PCR positives. Concurrent surveys, collected online through the REDCap platform, evaluated participant experiences of in-school swabbing.

Results 13988 swabs were collected from students and staff. There were zero positive test results for SARS-CoV-2, including no false positives. Participants reported high acceptability: $71 \%$ of students reported no or minimal discomfort and most were willing to be reswabbed (4\% refusal rate).

Conclusions $\mathrm{OP} / \mathrm{Na}$ swabbing is acceptable and repeatable in schoolchildren as young as 4 years old and may combat noncompliance rates by significantly increasing the acceptability of testing. This kind of minimally-invasive testing will be key to the success of ongoing, voluntary mass screening as society adjusts to a new 'normal' in the face of COVID-19.

Trial registration number Australian New Zealand Clinical Trials Registry-ACTRN12620000922976.

\section{Strengths and limitations of this study}

- Participation of 40 Western Australian schools, with broad representation across geography, socioeconomic demographics and school type.

- Minimally invasive SARS-CoV-2 swabbing method, likely to enhance rates of active consent and participation in COVID-19 screening.

- The sample size of this study is dictated by pragmatic, budgetary and logistical considerations.

- School selection was purposeful, not random, to ensure inclusion of a diverse sample.

\section{INTRODUCTION}

In late 2019, the SARS-CoV-2 virus emerged, and shortly thereafter, a global pandemic was declared. ${ }^{1}$ Governments responded with lockdown and isolation measures to combat the spread of COVID-19, including the closure of many schools. ${ }^{2}$ Quickly, it became clear that building capacity to test for COVID-19 rapidly and accurately would be critical for public safety and confidence in the reopening of schools. Here, we describe the results of the DETECT Schools Study, launched in Western Australia (WA), Australia, to trial a minimally invasive method for asymptomatic SARS-CoV-2 virus screening in primary and secondary schools across the state where children were swabbed unaccompanied by parents or caregivers.

The mandate of the DETECT Schools Study was simple: to screen asymptomatic students and staff swiftly and effectively for SARS-CoV-2 without causing discomfort. This speaks to a broader global need for transformative approaches to SARS-CoV-2 testing, as screening for the new virus becomes a part of daily life. As society grapples with a new 'normal', individuals with respiratory 
symptoms, those working in high-risk environments and those returning from travel are being swabbed regularly for SARS-CoV-2 in an effort to protect their communities.

At the onset of the COVID-19 pandemic, nasopharyngeal (NP) swabbing for PCR detection of SARS-CoV-2 was rapidly adopted globally as the gold standard for COVID-19 diagnosis; ${ }^{3}$ however, the validation of less invasive methods for virus detection is necessary to optimise compliance and increase the reach of mass screening programmes moving forwards.

At the time of this study, antigen tests were not yet available. Saliva sample PCR testing had emerged as a practical and non-invasive sampling method for the detection of SARS-CoV-2 in symptomatic ${ }^{4}$ and asymptomatic people, ${ }^{5}$ but there are conflicting studies concerning sensitivity, with some reporting similar detection rates to NP swabbing $^{6-8}$ while others indicate low sensitivity ${ }^{9}$ and caution against reliance on saliva samples alone for SARS-CoV-2 screening. ${ }^{10}$ Similarly, oropharyngeal (OP) swabbing is supported by some studies ${ }^{11}$ but displays inferior performance to NP swabbing in others. ${ }^{12}$ Nasal (Na) swabs offer another minimally invasive alternative with reasonable sensitivity, ${ }^{13}{ }^{14}$ which are found to be more sensitive than throat swabs for SARS-CoV-2 detection in children ${ }^{15}$ and are suited to high volume screening with a confirmatory NP swab. However, Na swabs collected late in the disease course are less sensitive than NP samples, ${ }^{16}$ and modelling suggests that this sampling technique in isolation does not effectively capture patients with a low viral load. ${ }^{17}$

$\mathrm{Na}$ swabbing has previously been found to be more comfortable and only marginally less sensitive than NP sampling for the detection of influenza. ${ }^{18}$ Pairing a Na swab with an OP swab offers a minimally invasive method for SARS-CoV-2 detection, with studies indicating specificity equivalent to and sensitivity marginally reduced $(\sim 3 \%)$ from that of NP swabbing. ${ }^{19-21}$ This sensitivity is reportedly retained when allowing self-collection ${ }^{22}$ or varying the swab type used ${ }^{23}$ Harnessing the sensitivity of both sampling techniques may maximise the chances of viral detection while remaining minimally invasive. So, does the use of OP/Na swabbing minimise discomfort enough to justify this small sacrifice in sensitivity? Here we report the use of $\mathrm{OP} / \mathrm{Na}$ swabbing to rapidly screen for SARS-CoV-2 in a large school-based cohort of volunteers, with an aim to optimise comfort and acceptability without losing sensitivity and specificity.

\section{METHODS}

The state of WA is vast, covering one-third the landmass of Australia. The population is concentrated in the capital city of Perth (2.1 million), with the remaining 400000 people spread across the State's 2.6 million square kilometres. There are 1131 schools across the state: 818 of these are public (Government) schools, at which a total of 315148 students were enrolled in $2020 .^{24}$

The study protocol is published. ${ }^{25}$ Briefly, 40 public schools (28 331 enrolled students and 4023 employed staff) were purposefully selected by the WA Department of Education for participation in the study, ensuring representative inclusion of education support schools, residential colleges and regional schools. Students aged 4-18 years were eligible, with two-thirds at metropolitan schools and one-third at regional schools from across the state (figure 1).

Prior to study commencement, written and video study and consent information were distributed by the schools to staff and parents, including study information and consent forms developed in consultation with a consumer advisory group and the Telethon Kids Institute Kulunga Aboriginal Research Development Unit. Staff and parents provided active informed consent through an online portal supported by the REDCap platform. ${ }^{26}$ Randomly selected consenting participants $(n=150 ; 90 \%$ students, $10 \%$ staff) were swabbed at each school in each round unless the school was not large enough to facilitate, in which case as many participants as possible were swabbed. Consented participants could subsequently refuse swabbing or withdraw from the study at any time.

SARS-CoV-2 swabbing of consented students and staff was carried out in the schools over three rounds between June and September 2020. We employed a combined OP and Na flocked swab (OP/Na) ${ }^{22}{ }^{23}$ During study development, swab comfort was investigated with a group of paediatric volunteers: the CITOSWAB flocked swab (Gaia Science, Singapore) was selected as the preferred swab for $\mathrm{OP} / \mathrm{Na}$ sampling.

Nurses received training in personal protective equipment (masks, gowns, eyewear and gloves) donning and doffing and swabbing technique before commencing the swabbing study in schools. Using a side-to-side motion, the swab was first swept across the back of the pharynx at least once in each direction, including both tonsils. The same swab was then inserted into one nostril (chosen by the child) along the floor of the Na cavity parallel to the palate until resistance was encountered, rotated gently five times, withdrawn, and placed in the sheath containing viral transport medium (CITOSWAB, Gaia Science, Singapore). Swabs were transported to the WA public laboratory service provider in Perth, WA, and tested for SARS-CoV-2 using an in-house PCR platform modified from the WHO recommended assay ${ }^{27}$ to include an inhibitor control, which detects the pan-sarbecovirus $\mathrm{E}$ gene. Validation studies of the PCR were performed early in the pandemic and confirmed a high analytical sensitivity and specificity with appropriate positive and negative controls. Any swab returning an in-house PCR positive result $(\mathrm{CT}$ value $<45$ ) was subject to confirmatory testing with the Xpert Xpress SARS-CoV-2 assay (Cepheid, California, USA). In-house and confirmatory PCR detections were reported as positive.

Surveys were administered to a subset of swabbing participants in the 2 weeks following the first round of swabbing in each school, and again a month after the completion of all swabbing rounds. Surveys asked about participant experiences of swabbing, including the level 


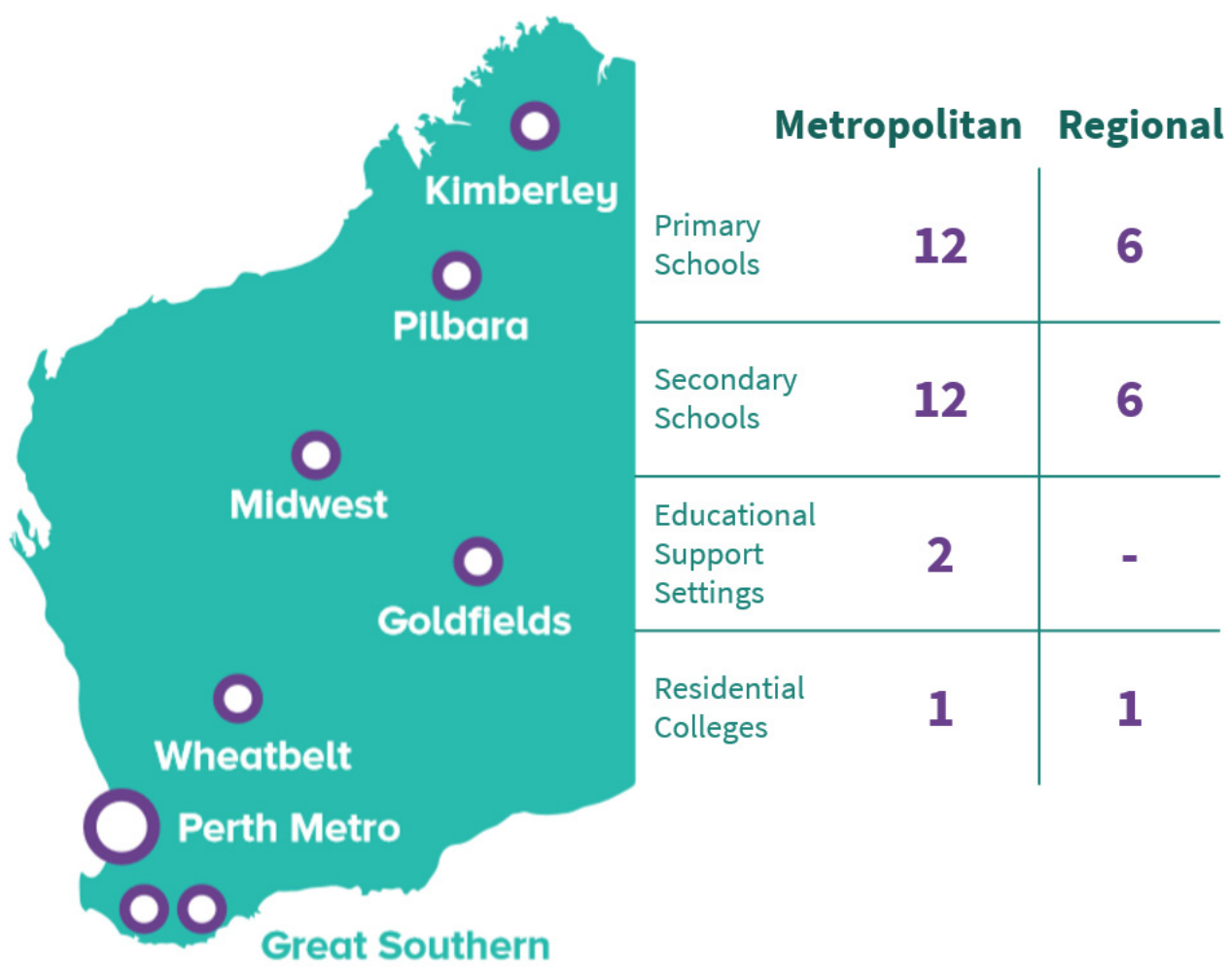

\section{Southwest}

Figure 1 Geographical distribution of schools participating in DETECT Schools Study swabbing.

of discomfort, concern and disruption associated with in-school testing. Parents were also surveyed about their child's swabbing experience. The surveys were administered during school classes for students and through personal email for staff and parents. Complete survey tools have been published previously. ${ }^{25}$

\section{Patient and public involvement}

Community involvement and advice was actively sought in the design and preparation of this study. Procedures and resources were reviewed and approved by a National Community Advisory Group for COVID-19 Research, convened by the Telethon Kids Institute and comprising community members from across Australia, including
Aboriginal members. The Telethon Kids Institute Kulunga Aboriginal Research Development Unit consulted on study resource development, including culturally-secure and informed consent processes and measures to support Aboriginal families.

\section{RESULTS}

One thousand four hundred fifty-eight school staff members and the parents of 7386 students engaged with the online consent platform. Seven thousand two hundred eight-one of these students (98.6\%) and 1321 staff $(90.6 \%)$ consented to be swabbed. Over the three

Table 1 Demographics of school students and staff participating in swabbing

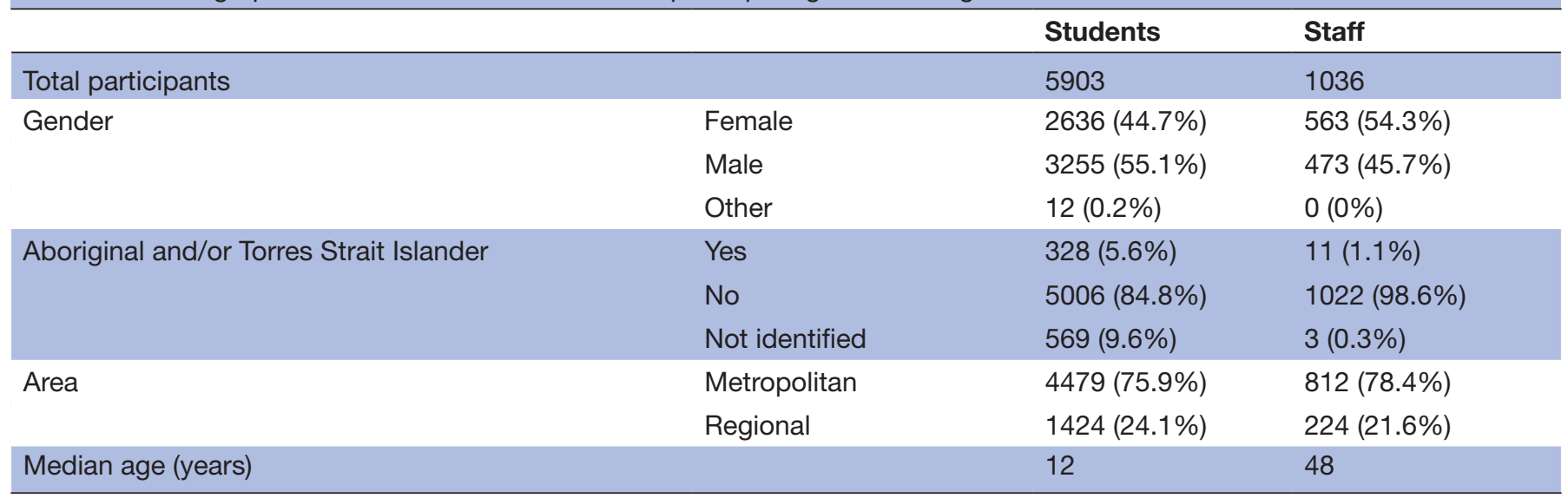


rounds, 13988 swabs were collected from 5903 students and 1036 staff (table 1).

Swabs were collected from across the state, and results provided by text message to all participating families and staff within 72 hours of sample collection. All but one sample returned negative results on the in-house PCR platform, and confirmatory Xpert testing of the in-house PCR detection returned a negative result. As such, none of the 13988 samples collected were positive for SARSCoV-2. This was consistent with no cases of local SARSCoV-2 transmission reported in WA throughout the study period.

Five thousand three hundred forty-nine students and 911 staff were randomised to be swabbed more than once across the three rounds. Of these participants, 214 students (4\%) and 12 staff $(1.3 \%)$ declined to be swabbed again (declined on the day or withdrew from the study).

After the first round of swabbing, the majority of student respondents indicated on a five-point scale (none, mild, moderate, painful, very painful) no more than mild discomfort (no discomfort: 19.7\%; mild discomfort: $51.0 \%$ ) (figure 2A). Most of the remaining students reported moderate discomfort (20.5\%), with few indicating that the swabbing was painful (painful: $6.5 \%$; very painful: $2.3 \%$ ). The majority of staff who had been swabbed also indicated only mild $(59.4 \%)$ or no $(19.6 \%)$ discomfort during the procedure.

Most students reported feeling only a little (37.2\%) or not at all $(47.3 \%)$ concerned about participating in testing (figure 2B). The parents of participating students also reported on their child's levels of concern, with the majority of parents observing little $(28.4 \%)$ or no $(60.8 \%)$ concern in their children.

Participating students were also asked whether they had been concerned about swabbing nurses wearing personal protective equipment at their school. For the most part, students reported only a little or no concern about this. Primary school students were slightly more likely to be at least moderately concerned $(10 \%)$ than secondary students (5\%) (figure 2C).

After three rounds of swabbing, surveys were administered again to an unmatched subset of swabbing participants. Response distributions were comparable to those described for the first survey cycle, with the majority of those surveyed still indicating mild levels of discomfort and concern after ongoing testing.

\section{DISCUSSION}

Efficient, accurate SARS-CoV-2 screening will be key to ameliorating the progression of the COVID-19 pandemic. As epidemiological evidence suggests that asymptomatic and presymptomatic individuals play a significant role in propagating the transmission of the virus, ${ }^{28-30}$ in low prevalence settings like WA the screening of asymptomatic populations will continue to be important to prevent a rise in cases. Without the indication of symptoms, this mode of screening requires good will and voluntary participation and must therefore strike a balance between optimising both testing sensitivity and participant comfort. As the discomfort associated with NP SARS-CoV-2 swabbing techniques risks poor adherence to mass screening campaigns, ${ }^{31}$ alternative approaches will be necessary to cultivate the consistency and reliability of public swabbing adherence necessary moving forwards.

For school-aged children, closing schools to combat the spread of COVID-19 must be balanced against the very real challenges in mental health and inequality likely associated with missing out on the educational and social benefits of school attendance. ${ }^{32} 33$ Consequently, countries around the world have mobilised to implement mass testing in an effort to support the reopening of schools and other establishments. COVID-19 molecular surveillance will be important moving forwards to ensure the safety of schools and individuals, especially in high prevalence countries in which cases continue to climb. Refinement of a robust and well-accepted screening mechanism is required to support the continuation of education; however very little data has been available on the acceptability of various swabbing procedures and how this may impact adherence to screening programmes.

Through the DETECT Schools Study we have evaluated the acceptability of $\mathrm{OP} / \mathrm{Na}$ sampling, reported to facilitate SARS-CoV-2 detection with limited or no sacrifice in sensitivity compared with the standard NP procedure, ${ }^{1834}$ in a school setting. Sampling was conducted with a flocked nylon swab: while evidence suggests that cotton, synthetic, flocked and non-flocked swabs all exhibit comparable performance for SARS-CoV-2 detection, ${ }^{35}$ flocked swabs have previously been shown to deliver a higher yield when swabbing for other respiratory viruses. ${ }^{36}$

In a large, representative cohort of school students and staff, our findings indicate that the vast majority of participants experienced minimal or no discomfort during an $\mathrm{OP} / \mathrm{Na}$ swab. Almost all of those who were asked to participate a second time agreed, illustrating the high tolerance for repeat procedures which is desirable for optimised respiratory screening programmes. This also suggests that individuals may be open to completing self-collected sampling, which has been shown to deliver adequate sensitivity for SARS-CoV-2 detection. ${ }^{37}$ Decreased discomfort is also likely to be associated with a reduced possibility of coughing, gagging or sneezing during sampling, in turn decreasing the risk of viral exposure for healthcare staff. While potentially not acceptable in specific settings with vulnerable groups for which sensitivity is paramount, such as entry screening for nursing homes, ${ }^{38}$ we argue that in schools and other similar settings this small decrease in sensitivity is far outweighed by high rates of consent and compliance which will allow for widespread testing.

This study was part of WA's jurisdictional response to the COVID-19 pandemic in April 2020. At the time of design, the state had been in a complete lockdown for 5 weeks, and schools were closed. The study was designed and implemented to reassure families and the public that schools could reopen, and to inform the level of risk of 
A)

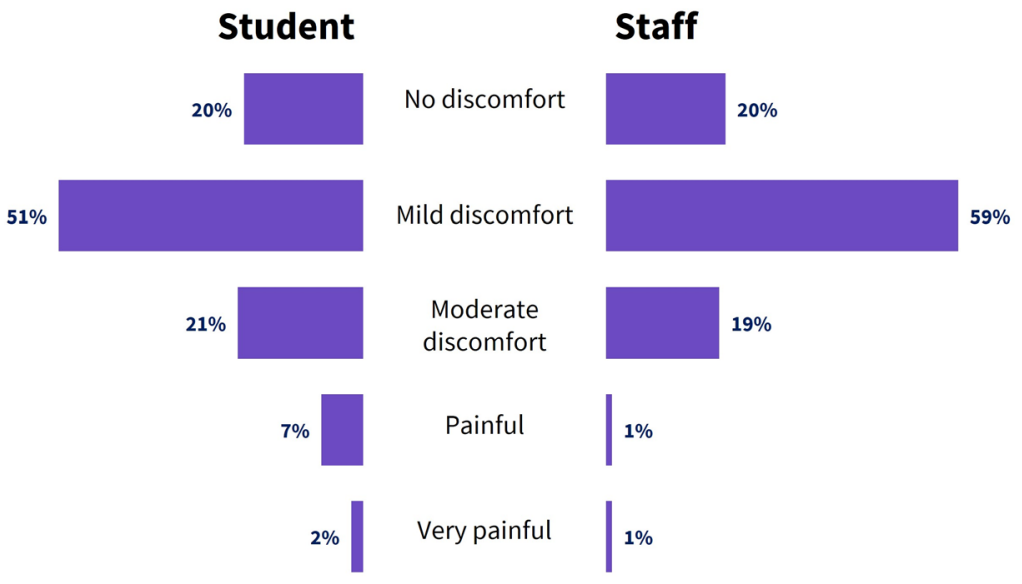

B)

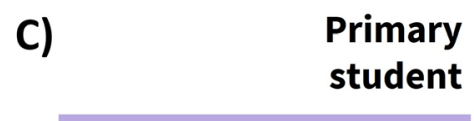

$69 \%$

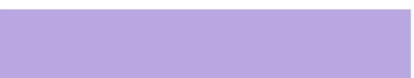

$21 \%$

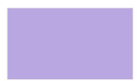

$6 \%$ Student
reported

$47 \%$

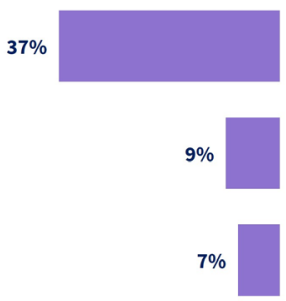

$0 \%$
A little concerned

\section{Parent}

reported

Not concerned
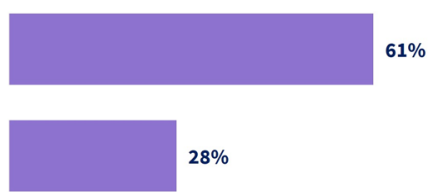

Moderately concerned

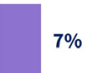

Quite concerned

$3 \%$

Extremely concerned

Figure 2 Distribution of survey responses regarding (A) self-reported discomfort (student and staff); (B) students' concern about being swabbed (self-reported and parent-reported); and (C) students' concern regarding swabbing staff use of PPE.

transmission in a school setting. However, during this period of time, transmission of SARS-CoV-2 was so well controlled with public health measures that there were no detected community cases of COVID-19 for almost 10 months and as such there were also no confirmed cases in the study. While this could be considered a methodological limitation, we have demonstrated the acceptability and ease of implementing a molecular based swabbing programme in a school context with minimal disruption to students or educational outcomes. 


\section{CONCLUSION}

Here we report an approach to large-scale asymptomatic swabbing for SARS-CoV-2 leading to high levels of willingness to participate. The sensitivity of this method for the identification of SARS-CoV-2 is supported by other studies. This methodology for screening children was well received by a large cohort and could be used to screen for asymptomatic SARS-CoV-2 in other settings, mitigating the requirements for uncomfortable NP sampling and leading to enhanced compliance with programmes designed to prevent onwards transmission of SARS-CoV-2.

\author{
Author affiliations \\ ${ }^{1}$ Telethon Kids Institute, Nedlands, Western Australia, Australia \\ ${ }^{2}$ Wesfarmers Centre of Vaccines \& Infectious Diseases, Telethon Kids Institute, \\ Nedlands, Western Australia, Australia \\ ${ }^{3}$ Edith Cowan University, Joondalup, Western Australia, Australia \\ ${ }^{4}$ University of Western Australia, Perth, Western Australia, Australia \\ ${ }^{5}$ Child and Adolescent Health Service, Perth, Western Australia, Australia \\ ${ }^{6}$ PathWest Laboratory Medicine Western Australia, Nedlands, Western Australia, \\ Australia \\ ${ }^{7}$ Curtin University, Perth, Western Australia, Australia
}

Acknowledgements We would like to acknowledge and thank staff from the WA Department of Education, PathWest, the WA Child and Adolescent Health Service and the WA Country Health Service for their valuable contribution to the DETECT Schools Study. We also thank all students and staff who participated in swabbing. Contributors HMT coordinated data collection, contributed to data analysis, conducted the literature search, generated figures and drafted the manuscript. MJM coordinated the conception and design of the study, coordinated data collection and provided critical revision of drafts. SA, TB, AL, AW coordinated data collection and provided critical revision of drafts. KL provided critical revision of drafts. MC, PGS, LL and DS contributed to data analysis and provided critical revision of drafts. AJM contributed to design of test workflow, data analysis and provided critical revision of drafts. AP and LS contributed to data collection and provided critical revision of drafts. JC, DC, PG and ACB oversaw conception, design and coordination of the study and provided critical revision of drafts. ACB, as guarantor, accepts full responsibility for the finished work and the conduct of the study, had access to the data, and controlled the decision to publish.

Funding This work was supported by the Western Australian Department of Health [DoH20205875]. Donna Cross' contribution to this paper was supported by a National Health and Medical Research Council Research Fellowship [GNT1119339]. ACB receives an Investigator Award from the National Health and Medical Research Council of Australia [GNT1175509]. PG's contribution to this paper was supported by Channel 7 Telethon Trust, Western Australia.

Competing interests Telethon Kids Institute authors report grants from the Western Australian Department of Health during the conduct of this study. DC and ACB report grants from the Western Australian Department of Health outside the submitted work. ACB, AP, LS, DS and AJM are employees of the Western Australian Department of Health.

Patient and public involvement Patients and/or the public were involved in the design, or conduct, or reporting, or dissemination plans of this research. Refer to the Methods section for further details.

\section{Patient consent for publication Not applicable.}

Ethics approval Ethical approval was obtained from the WA Child and Adolescent Health Service (PRN RGS0000004059) and the WA Aboriginal Health Ethics Committee (PRN 993)

Provenance and peer review Not commissioned; externally peer reviewed.

Data availability statement Data are available upon reasonable request. DETECT Schools Study deidentified participant data are shared with study partners (WA Departments of Health and Education) but are not available to the public. The study protocol is published at https://doi.org/10.3389/fpubh.2021.636921https://doi.org/ 10.3389/fpubh.2021.636921.

Open access This is an open access article distributed in accordance with the Creative Commons Attribution Non Commercial (CC BY-NC 4.0) license, which permits others to distribute, remix, adapt, build upon this work non-commercially, and license their derivative works on different terms, provided the original work is properly cited, appropriate credit is given, any changes made indicated, and the use is non-commercial. See: http://creativecommons.org/licenses/by-nc/4.0/.

ORCID iD

Hannah M Thomas http://orcid.org/0000-0003-3699-7714

\section{REFERENCES}

1 Du Toit A. Outbreak of a novel coronavirus. Nat Rev Microbiol 2020;18:123.

2 Viner RM, Russell SJ, Croker H, et al. School closure and management practices during coronavirus outbreaks including COVID-19: a rapid systematic review. Lancet Child Adolesc Health 2020;4:397-404.

3 World Health Organisation. Laboratory testing for coronavirus disease 2019 (COVID-19) in suspected human cases: interim guidance 2020

4 Azzi L, Carcano G, Gianfagna F, et al. Saliva is a reliable tool to detect SARS-CoV-2. J Infect 2020;81:e45-50.

5 Yokota I, Shane PY, Okada K, et al. Mass screening of asymptomatic persons for SARS-CoV-2 using saliva. SSRN Electronic Journal 2020.

6 Vaz SN, Santana DSde, Netto EM, et al. Saliva is a reliable, noninvasive specimen for SARS-CoV-2 detection. Braz $J$ Infect Dis 2020;24:422-7.

7 KK-W T, Tsang OT-Y, CC-Y Y, et al. Consistent detection of 2019 novel coronavirus in saliva. Clin Infect Dis an Off Publ Infect Dis Soc Am 2020;71:841-3.

8 Chen JH-K, Yip CC-Y, Poon RW-S, et al. Evaluating the use of posterior oropharyngeal saliva in a point-of-care assay for the detection of SARS-CoV-2. Emerg Microbes Infect 2020;9:1356-9.

9 Lin C, Xiang J, Yan M, et al. Comparison of throat swabs and sputum specimens for viral nucleic acid detection in 52 cases of novel coronavirus (SARS-Cov-2)-infected pneumonia (COVID-19). Clin Chem Lab Med 2020;58:1089-94.

10 Riccò M, Ranzieri S, Peruzzi S, et al. RT-qPCR assays based on saliva rather than on nasopharyngeal swabs are possible but should be interpreted with caution: results from a systematic review and meta-analysis. Acta Biomed 2020;91:e2020025.

11 Calame A, Mazza L, Renzoni A, et al. Sensitivity of nasopharyngeal, oropharyngeal, and nasal wash specimens for SARS-CoV-2 detection in the setting of sampling device shortage. Eur J Clin Microbiol Infect Dis 2021;40:441-5.

12 Wang X, Tan L, Wang X, et al. Comparison of nasopharyngeal and oropharyngeal swabs for SARS-CoV-2 detection in 353 patients received tests with both specimens simultaneously. Int J Infect Dis 2020;94:107-9.

13 Tu Y-P, Jennings R, Hart B, et al. Swabs collected by patients or health care workers for SARS-CoV-2 testing. N Engl J Med 2020;383:494-6.

14 McCulloch DJ, Kim AE, Wilcox NC, et al. Comparison of unsupervised home self-collected Midnasal swabs with cliniciancollected nasopharyngeal swabs for detection of SARS-CoV-2 infection. JAMA Netw Open 2020;3:e2016382.

15 Palmas G, Moriondo M, Trapani S, et al. Nasal swab as preferred clinical specimen for COVID-19 testing in children. Pediatr Infect Dis J 2020;39:e267-70.

16 Pinninti S, Trieu C, Pati SK, et al. Comparing nasopharyngeal and midturbinate nasal swab testing for the identification of severe acute respiratory syndrome coronavirus 2. Clin Infect Dis 2021:72:1253-5.

17 Callahan C, Lee R, Lee G, et al. Nasal-Swab testing misses patients with low SARS-CoV-2 viral loads. medRxiv 2020. doi:10.1101/2020.0 6.12.20128736. [Epub ahead of print: 14 Jun 2020].

18 Frazee BW, Rodríguez-Hoces de la Guardia A, Alter $\mathrm{H}$, et al. Accuracy and discomfort of different types of intranasal specimen collection methods for molecular influenza testing in emergency department patients. Ann Emerg Med 2018;71:509-17.

19 LeBlanc JJ, Heinstein C, MacDonald J, et al. A combined oropharyngeal/nares swab is a suitable alternative to nasopharyngeal swabs for the detection of SARS-CoV-2. J Clin Virol 2020;128:104442.

20 Vlek ALM, Wesselius TS, Achterberg R, et al. Combined throat/nasal swab sampling for SARS-CoV-2 is equivalent to nasopharyngeal sampling. Eur J Clin Microbiol Infect Dis 2021;40:1-3.

21 Tsang NNY, So HC, Ng KY, et al. Diagnostic performance of different sampling approaches for SARS-CoV-2 RT-PCR testing: a systematic review and meta-analysis. Lancet Infect Dis 2021;21:1233-45. 
22 Wehrhahn MC, Robson J, Brown S, et al. Self-Collection: an appropriate alternative during the SARS-CoV-2 pandemic. J Clin Virol 2020;128:104417.

23 Patriquin G, Davis I, Heinstein C, et al. Exploring alternative swabs for use in SARS-CoV-2 detection from the oropharynx and anterior nares. J Virol Methods 2020;285:113948.

24 Western Australian Department of Education. Public and nongovernment schools with full-time students (by metropolitan, country and combined) - Semester 2, 2020. School Information - Statistical Reports 2020.

25 Mullane MJ, Thomas HM, Epstein M, et al. Detect schools study protocol: a prospective observational cohort surveillance study investigating the impact of COVID-19 in Western Australian schools. Front Public Health 2021;9:16.

26 Harris PA, Taylor R, Thielke R, et al. Research electronic data capture (REDCap)-a metadata-driven methodology and workflow process for providing translational research informatics support. J Biomed Inform 2009;42:377-81.

27 Corman VM, Landt O, Kaiser M, et al. Detection of 2019 novel coronavirus (2019-nCoV) by real-time RT-PCR. Eurosurveillance 2020;25.

28 Bai Y, Yao L, Wei T, et al. Presumed asymptomatic carrier transmission of COVID-19. JAMA 2020;323:1406-7.

29 Mizumoto K, Kagaya K, Zarebski A, et al. Estimating the asymptomatic proportion of coronavirus disease 2019 (COVID-19) cases on board the diamond Princess cruise SHIP, Yokohama, Japan, 2020. Eurosurveillance 2020;25.
30 Moghadas SM, Fitzpatrick MC, Sah P, et al. The implications of silent transmission for the control of COVID-19 outbreaks. Proc Natl Acad Sci U S A 2020;117:17513-5.

31 Moisset X, Gautier N, Godet T, et al. Nasopharyngeal swab-induced pain for SARS-CoV-2 screening: a randomised controlled trial of conventional and self-swabbing. Eur J Pain 2021;25:924-9.

32 Armitage R, Nellums LB. Considering inequalities in the school closure response to COVID-19. Lancet Glob Health 2020;8:e644.

33 Lee J. Mental health effects of school closures during COVID-19. The Lancet Child \& Adolescent Health 2020;4:421.

34 Jamal AJ, Mozafarihashjin M, Coomes E, et al. Sensitivity of midturbinate versus nasopharyngeal swabs for the detection of severe acute respiratory syndrome coronavirus 2 (SARS-CoV-2). Infect Control Hosp Epidemiol 2021;42:1001-3.

35 Garnett L, Bello A, Tran KN, et al. Comparison analysis of different swabs and transport mediums suitable for SARS-CoV-2 testing following shortages. J Virol Methods 2020;285:113947.

36 Moore C, Corden S, Sinha J, et al. Dry cotton or flocked respiratory swabs as a simple collection technique for the molecular detection of respiratory viruses using real-time NASBA. $J$ Virol Methods 2008;153:84-9.

37 Braz-Silva PH, Mamana AC, Romano CM, et al. Performance of at-home self-collected saliva and nasal-oropharyngeal swabs in the surveillance of COVID-19. J Oral Microbiol 2020;13:1858.

38 Mina MJ, Andersen KG. COVID-19 testing: one size does not fit all. Science 2021;371:126-7. 